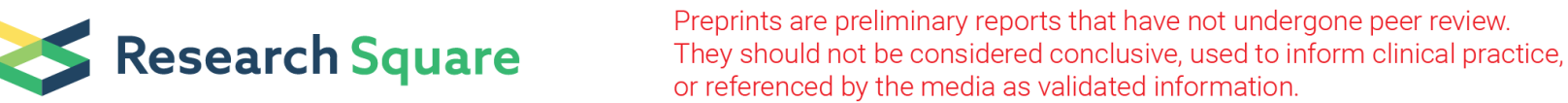

\section{Effect of Phenolic Acids on Temperature-sensitive Property of Self-assembly of Ionic Pair of Poly(ethylene imine)/(phenylthio)Acetic Acid}

\section{Fanyu Zhao}

Kangwon National University

Jin-Chul Kim ( $\nabla$ jinkim@kangwon.ac.kr)

Kangwon National University https://orcid.org/0000-0003-3717-6096

\section{Research}

Keywords: Ionic pair, Self-assembly, Phenolic acid, Upper critical solution temperature, Temperatureresponsive release

Posted Date: September 22nd, 2021

DOI: https://doi.org/10.21203/rs.3.rs-907833/v1

License: (c) (i) This work is licensed under a Creative Commons Attribution 4.0 International License. Read Full License 


\section{Abstract}

The ionic pair self-assembly (IPSAM) composed of poly(ethyleneimine) (PEI) and (phenylthio)acetic acid (PTA) was prepared and the effect of phenolic acids (PAs) (e.g. cinnamic acid (CA), hydroxycinnamic acid (HCA), and dihydroxycinnamic acid (DHCA)) on the upper critical solution temperature (UCST) and the temperature-responsive releasing property of IPSAM were investigated. PEI/PTA ionic pair showed a UCST behavior and the PAs decreased the UCST effective in the order of DHCA > HCA > CA. The PAs were thought to attach to the PEI chain of PEI/PTA ionic pair as pendants. According to the interfacial tensiometry, PEI/PTA(3/7) ionic pair was found to be air/water interface-active due to their amphiphilic property. CA had little effect on the interfacial activity of the ionic pair. HCA and HDCA significantly decreased the interfacial activity possibly because they are more polar than CA thus the hydrophilic and lipophilic balance of PEI/PTA ionic pair could be broken by their attachment to PEI chains. IPSAM was found as nanoparticles whose diameter was tens of nanometer and PAs had little effect on the shape and the size of IPSAM, once the UCST of PEI/PTA/PA ionic pair was above room temperature where the TEM micrographs were taken. If the UCST of PEI/PTA/PA ionic pair was below room temperature, no particulate matters were found on the TEM micrographs. The release degree of cargo loaded in IPSAM increased slowly with time lapse below UCST. Whereas, the release degree increased rapidly with time lapse above UCST, possibly because of the thermally-induced disintegration of IPSAM.

\section{Introduction}

Self-assemblies are formed spontaneously when amphiphilic molecules come in contact with an aqueous solution [1], [2]. The second law of thermodynamics (free energy-minimizing process) accounts for the self-assembling phenomena. Since an amphiphilic molecule acts as a building block and it constitutes the assembly, the shape of the building block can determine the structure of the self-assembly according to a space-filling model [3], [4]. Packing parameter $(P)$ reflects the shape of the amphiphilic molecules and provides an intuition about what they would be assembled into [5], [6], [7]. For example, an amphiphilic molecule whose packing parameter is close to 1 is almost rectangular and is likely to be assembled into vesicular bilayers. An amphiphilic molecule whose packing parameter deviates much from 1 is either conical or reversed conical and it can form a micelle or hexagonal phase. The selfassemblies are versatile as drug carriers because they can be easily prepared due to their self-assembling property, they can load hydrophilic drugs and lipophilic ones in their polar and non-polar domain, and they can penetrate into tissues effectively due to the small size. In addition, they can be made to release their contents in response to stimuli including $\mathrm{pH}$ value, temperature, redox, electric field, magnetic field, etc. [8-16]. Recently, the ionic pair of poly(ethylene imine) (PEI) and cinnamic acid (CA) was reported to form self-assemblies [17], [18]. CA molecules can be conjugated to PEI chains through the electrostatic interaction between the carboxylic group and the amino group. Since PEI chains are polar and the phenyl groups of CA are non-polar, the ionic pair of PEI and CA was claimed to be amphiphilic and it could be assembled into nanospheres in an aqueous solution. The ionic pair of PEI and CA exhibited an upper critical solution temperature (UCST) and the resulting assembly released its content in response to 
change in temperature, change in $\mathrm{pH}$ value, and UV irradiation. More recently, a self-assembly that was responsive to temperature and oxidation was developed by taking advantage of the ionic pair of PEI and (phenylthio)acetic acid (PTA) [19]. PTA is a sulfide compound, it has a hydrophobic phenyl group and a carboxyl group in its structure, and it can ionically interact with PEI. The ionic pair of PEI/PTA was selfassembled into nanoparticles in an aqueous phase due to its amphiphilic property and showed a UCST behavior. The self-assembly of the ionic pair released its cargo in answering to a temperature increase, possibly because, as the temperature increases, PTA becomes solubilized, the ionic pair loses its amphiphilic property, and the assembly is disassembled. In addition, the self-assembly was oxidationsensitive in terms of release. Once the sulfide of PTA has been oxidized to sulfoxide and sulfone, it becomes more polar and the ionic pair is likely to lose its amphiphilic property, leading to the disintegration of the self-assembly.

In this study, phenolic acids (PAs) were included in the self-assembly of PEI/PTA ionic pair to investigate their effect on the temperature-dependent self-assembling property. Cinnamic acid (CA), hydroxycinnamic acid (HCA), dihydroxy cinnamic acid (DHCA) were used as PAs. Phenolic acid (PA) has a carboxylic group thus it can compete with PTA in binding to PEl chains. Since the polarity of the PAs is obviously less than that of PTA, the PAs would be able to lower the UCST of PEI/PTA ionic pair. Accordingly, the thermally induced-disintegration of the self-assembly thus the release of cargo loaded in the self-assembly would readily be promoted by including a phenolic acid in PEI/PTA ionic pair (Graphical Abstract).

\section{Experimental}

\subsection{Materials}

Poly(ethylene imine) (PEl, branched, average MW: 2000), trizma base, cinnamic acid (CA), and $N, N-$ dimethylformamide were purchased from Sigma-Aldrich Co. (MO, USA). (Phenylthio)acetic acid (PTA), dihydroxycinnamic acid (DHCA), and Nile red were purchased from Tokyo Chemical Industry Co., Ltd. (Tokyo, Japan). Hydroxycinnamic acid (HCA) was purchased from Alfa aesar. Water was doubly distilled in a Milli-Q water purification system (Millipore Corp, MA, USA) until the resistivity was $18 \mathrm{M} \Omega / \mathrm{cm}$. All other reagents were in analytical grade.

\subsection{Investigation of PEI/PTA ionic pairing by FT-IR spectroscopy}

Different amounts of PEl was dissolved in Trizma buffer (30mM, pH7.0) so that the concentration was $3.7 \mathrm{mg} / \mathrm{ml}, 2.8 \mathrm{mg} / \mathrm{ml}, 2 \mathrm{mg} / \mathrm{ml}, 1.5 \mathrm{mg} / \mathrm{ml}$, and $1 \mathrm{mg} / \mathrm{ml}$. Then, variable amounts of PTA were put into a vial containing the PEl solution so that the concentration of PTA was $6.3,7.2,8.0,8.5$, and $9 \mathrm{mg} / \mathrm{ml}$ and the molar ratio of the amino group of PEl to the carboxyl group of PTA was $7: 3,6: 4,5: 5,4: 6$, and 3:7. The total concentration of PEI plus PTA was $10 \mathrm{mg} / \mathrm{ml}$. In case the molar ratio of the amino group to the carboxyl group was $a: b$, the mixture suspension was abbreviated to PEI/PTA(a/b). After freeze-drying the mixture suspensions, each of dry PEI/PTA(a/b), PEI, and PTA was mixed with $\mathrm{KBr}$ and homogenized in a 
mortar using a pestle. The pellets of the mixture powders were prepared by putting the powders in a mold and pressing them using a press. The FT-IR spectra were obtained on a Fourier Transformed Infrared Spectrophotometer (FT-IR, FT-3000-Excalibur, Varian Inc., CA, USA).

\subsection{Investigation of upper critical solution temperature behavior}

PEI/PTA(3/7) whose weight ratio of PEI to PTA was 1:9 was put into a vial. Each of CA, HCA, DHCA was added to the PEI/PTA mixture suspension so that the molar ratio of the amino group of PEl/the carboxyl group of PTA/the carboxyl group of PA was 3:7:1.4, 3:7:4.2, and 3:7:7. The mixture suspensions of PEI/PTA/PA were agitated by rolling the vials on a roller mixer at room temperature for $24 \mathrm{~h}$. PEI/PTA/PA mixture whose molar ratio of the amino group of PEI/the carboxyl group of PTA/the carboxyl group of PA was a:b:c was termed as PEI/PTA/PA(a/b/c). The optical density at $600 \mathrm{~nm}$ of the suspensions was recorded on a UV spectrophotometer (UK 6505 UV/Vis Spectrophotometer, JENWAY, UK) while heated at a rate of $2{ }^{\circ} \mathrm{C} / \mathrm{min}$ from 20 to $50^{\circ} \mathrm{C}$ using a temperature controller (CW-05G, Jeio Tech, South Korea). A line was fitted to the data points on plateau region and another line to those on increasing region. $\mathrm{A}$ temperature corresponding to the intersection of two lines was defined as an upper critical solution temperature.

\subsection{Investigation of interfacial tension}

The interfacial tension of PEI/PTA/PA(a/b/c) suspension was determined by a ring method. $\mathrm{PEI} / \mathrm{PTA} / \mathrm{PA}(\mathrm{a} / \mathrm{b} / \mathrm{c})$ suspension whose PEI plus PTA concentration was $10 \mathrm{mg} / \mathrm{ml}$ was prepared under the same condition as described in the section "2.3.". PEI/PTA/PA(a/b/c) suspension was sequentially diluted using Trizma buffer solution ( $30 \mathrm{mM}, \mathrm{pH} 7.0)$ to obtain different concentrations. Each of PEl and PTA solution, of which PEI and PTA concentration were the same as those of PEI/PTA/PA(a/b/c) suspension, was prepared as control and sequentially diluted with the same buffer solution. An interfacial tensiometer (DST 60, SEO Co., Suwon, South Korea) was exploited to measure the interfacial tension.

\subsection{Transmission electron microscopy}

All these different ionic pair self-assemblies of PEI/PTA (IPSAMs) with/without PAs at different molar ratios were negatively stained by mixing the IPSAM suspensions and a uranyl acetate solution $(1.2 \%$ $(\mathrm{w} / \mathrm{v}))$ in 1:1 (v/v) ratio and keeping the mixture suspension at room temperature under dark condition for $5 \mathrm{~h}$. A drop of mixture suspension was transferred onto the surface of a formvar/copper-coated grid and it was air-dried overnight under dust-free condition. The TEM micrograph of IPSAMS was obtained using a transmission electron microscopy (LEO-912AB OMEGA, LEO, Germany), installed at Korea Basic Science Institute (KBSI, located in Chuncheon, Republic of Korea)).

\subsection{Observation of PEI/PTA interaction by ${ }^{1} \mathrm{H}$ NMR spectroscopy}


To understand how PA could interact with the PTA of PEI/PTA ionic pair, ${ }^{1} \mathrm{H}-\mathrm{NMR}$ spectroscopy was carried out at room temperature. PEI/PTA(3/7) and PEI/PTA/PA(3/7/1.4) suspension were prepared as described in a previous section except that $\mathrm{D}_{2} \mathrm{O}$ was used as a solvent instead of the buffer solution. In parallel, each of PA, PEI, and PTA was dissolved in $\mathrm{D}_{2} \mathrm{O}$ to prepare reference solutions. ${ }^{1} \mathrm{H}$ NMR spectrum was taken on a Bruker Avance $400 \mathrm{MHz}$ spectrometer (Karlsruhe, Germany, installed in the Central Laboratory Center of Kangwon National University).

\subsection{Temperature-responsive release}

$100 \mu \mathrm{l}$ of Nile red solution ( $1 \mathrm{mg} / \mathrm{ml}$, in DMF) was added to $10 \mathrm{ml}$ each of PEI/PTA(3/7) and PEI/PTA/PA(3/7/1.4) suspension (10mg PEl plus PTA/ml, in Trizma buffer (30 mM, pH 7.0)) contained in a $20 \mathrm{ml}$ vial and the mixture suspensions were agitated at room temperature overnight. $2 \mathrm{ml}$ of the dyeloaded IPSAM suspension was put into a cuvette and it was placed in a holder thermostated at a specific temperature (i.e. $24,30,37$, and $45^{\circ} \mathrm{C}$ ). The fluorescence intensity was determined at $615 \mathrm{~nm}$ for $8 \mathrm{~min}$ on a fluorescence spectrophotometer (Hitachi F2500, Hitachi, Japan) while being exited at $570 \mathrm{~nm}$.

Release \% was determined by the following equation reported elsewhere [20, 21].

Release $\%=\left(1-F_{t} / F_{0}\right) \times 100 \%$

Where, $F_{t}$ is the fluorescence intensity measured at a specific time lapse, and $F_{0}$ is the fluorescence intensity measured when the time lapse is zero.

\section{Results}

\subsection{Investigation of PEI/PTA ionic pairing by FT-IR spectroscopy}

Figure 1 shows the FT-IR spectrum of PEI, PTA, and dry PEI/PTA(a/b). In the spectrum of PEI, the signal of the amino group was found around $3300 \mathrm{~cm}^{-1}$. In the spectrum of PTA, the signal of the carbonyl group was found around $1700 \mathrm{~cm}^{-1}$. In the spectrum of dry PEI/PTA(a/b), the signal of the amino group (around $3300 \mathrm{~cm}^{-1}$ ) was very weak and hardly found and the signal of the carbonyl group (around $1700 \mathrm{~cm}^{-1}$ ) was significantly weaker than that of PTA alone, even disappeared for dry PEI/PTA(7/3). Besides, two signals were newly found at $1573 \mathrm{~cm}^{-1}$ and $1406 \mathrm{~cm}^{-1}$, corresponding to carboxylate (COO ). These indicated that the carboxyl group and the amino group were attracted to each other, forming PEI/PTA ionic pairs.

\subsection{Investigation of upper critical solution temperature behavior}


Figure $2(\mathrm{~A})$ shows the transmittance of the PEI/PTA/CA mixture suspensions with increasing temperature. PEI/PTA/CA(3:7:0) suspension was turbid and exhibited almost $0 \%$ transmittance until the temperature reached $40^{\circ} \mathrm{C}$. PEI/PTA/CA(3:7:0) ionic pair can be self-assembled in an aqueous phase owing to its amphiphilic property and the ionic pair self-assembly (IPSAM) scatters the visible light, leading to almost $0 \%$ transmittance. The transmittance began to increase around $46^{\circ} \mathrm{C}$ and it increased with increasing temperature. As the temperature increases, PTA would become solubilized more readily in aqueous phase, PEI/PTA/CA(3:7:0) ionic pair would decrease in its amphiphilicity, and the IPSAM was likely to be dissembled, resulting in an increase in the transmittance. Based on the intersection of two tangential lines, the UCST of PEI/PTA/CA(3:7:0) ionic pair was estimated to be about $48^{\circ} \mathrm{C}$.

The transmittance of PEI/PTA/CA(3:7:1.4) suspension changed with temperature in a similar manner to that of PEI/PTA/CA(3:7:0) suspension, thus it could be said that PEI/PTA/CA(3:7:1.4) ionic pair also exhibited UCST behavior. The UCST of PEI/PTA/CA(3:7:1.4) ionic pair was evaluated to be about $45^{\circ} \mathrm{C}$ and it was about $3^{\circ} \mathrm{C}$ lower than that of PEI/PTA/CA(3:7:0) ionic pair.

The pKa of the carboxyl group of PTA was reported to be 3.7, the pKa of CA is known to be 4.44, and the pKb of the amino group of PEl was assumed to be 9.1 [22-24]. Using the Henderson-Hasselbalch equation, the ionization degree at $\mathrm{pH} 7.0$ of the amino group of PEl, that of the carboxyl group of PTA, and that of the carboxyl group of CA were calculated to be $99.2 \%, 99.7 \%$, and $99.7 \%$, suggesting that most of the amino groups and carboxylic groups were ionized in the medium (i.e. Trizma buffer $(30 \mathrm{mM}$, $\mathrm{pH} 7.0)$ ).

If the amino group of PEl ionically bonds to the carboxyl group of PTA in 1:1 ratio, PTA is excessive, compared with PEI, and the \% excess was calculated to be about 133. The excessive amount of PTA molecules were thought to bind to PTA ones that was electrostatically bound to PEl chains through hydrophobic interaction among the phenyl groups.

Likewise, CA molecules would be able to bind to PTA molecules attached to PEI chains through the hydrophobic interaction. Alternatively, CA molecule would compete with PTA in binding to PEI chains ionically. Either way, CA seemed to bind to PEI chains, giving a rise to the change of UCST. CA has a double bond in its structure but PTA dose not. Since a double bond is more polar than a single bond, CA would be more polar than PTA [25]. If CA is bound to PEI/PTA ionic pair, the IPSAM is likely to be disassembled at a lower temperature due to its higher polarity. This would account for why the UCST of PEI/PTA/CA(3/7/1.4) ionic pair was lower than that of PEI/PTA/CA(3/7/0) ionic pair.

When CA was included more, the UCST was found at a much lower temperature. For example, the UCST of PEI/PTA/CA(3/7/4.2) ionic pair was evaluated to be about $34^{\circ} \mathrm{C}$ and it was about $9{ }^{\circ} \mathrm{C}$ lower than that of PEI/PTA/CA(3/7/1.4). Compared with PEI/PTA/CA(3/7/1.4) ionic pair, PEI/PTA/CA(3/7/4.2) ionic pair had more $C A$ in its structure thus it would be more susceptible to temperature increase due to the higher polarity of CA. Further increase in the content of CA reduced the UCST but not as much as previously. For example, the UCST of PEI/PTA/CA(3/7/7) ionic pair was found to be about $33^{\circ} \mathrm{C}$ and it was only about 
$1{ }^{\circ} \mathrm{C}$ lower than that of PEI/PTA/CA(3/7/4.2). The amount of CA of PEI/PTA/CA(3/7/7) ionic pair seemed to be high enough for the CA effect on the UCST to be saturated.

Figure 2 (B) shows the transmittance of the PEI/PTA/HCA mixture suspensions with increasing temperature. The transmittance of PEI/PTA/HCA(3:7:1.4) suspension began to increase around $34^{\circ} \mathrm{C}$ and reached almost $100 \%$ when the temperature increased to $50^{\circ} \mathrm{C}$. It could be said that, like PEI/PTA/CA(3:7:1.4) ionic pair, PEI/PTA/HCA(3:7:1.4) ionic pair showed UCST behavior. Based on the intersection of two tangential lines, the UCST of PEI/PTA/HCA(3:7:1.4) ionic pair was evaluated to be about $39^{\circ} \mathrm{C}$. The UCST of PEI/PTA/HCA $(3: 7: 1.4)$ ionic pair was about $9{ }^{\circ} \mathrm{C}$ lower than that of PEI/PTA(3:7) ionic pair (about $48^{\circ} \mathrm{C}$ ). Like CA, HCA would be able to bind to the PTA of PEI/PTA ionic pair through the hydrophobic interaction and to the PEI chain via electrostatic interaction. If HCA is bound to PEI/PTA (3/7) ionic pair either way, the IPSAM is likely to be disassembled at a lower temperature because the hydrophobic part of HCA (hydroxyl phenyl group) is more polar than that of PTA (phenyl group).

HCA was more effective than CA in decreasing the UCST of PEI/PTA(3/7) ionic pair. For example, The UCST of PEI/PTA/HCA(3:7:1.4) ionic pair was about $6^{\circ} \mathrm{C}$ lower than that of PEI/PTA/CA(3:7:1.4) ionic pair (about $45^{\circ} \mathrm{C}$ ). The hydrophobic part of HCA (hydroxyl phenyl group) is more polar than that of $\mathrm{CA}$ (phenyl group) $[25,26]$. This would account for why HCA decreased the UCST more effectively than CA.

The UCST decreased furthermore when the HCA content increased. For example, the UCST of $\mathrm{PEI} / \mathrm{PTA} / \mathrm{HCA}(3 / 7 / 4.2)$ ionic pair seemed to be below $20^{\circ} \mathrm{C}$ and the that of PEI/PTA/HCA(3/7/7) ionic pair was not found in the temperature window possibly because its UCST was far below $20^{\circ} \mathrm{C}$. As the HCA content is higher, it can be accumulated more as the pendants of the PEI chains, the pendants are likely to become more polar, and the IPSAM would be disassembled at a lower temperature.

Figure 2 (C) shows the transmittance of the PEI/PTA/DHCA mixture suspensions with increasing temperature. The transmittance of PEI/PTA/DHCA(3:7:1.4) suspension increased with the temperature in a similar manner to that of PEI/PTA/HCA(3:7:1.4) suspension. By drawing a tangential line, the UCST of PEI/PTA/DHCA(3:7:1.4) ionic pair was estimated to be about $27^{\circ} \mathrm{C}$. PEI/PTA/DHCA(3:7:1.4) ionic pair exhibited its UCST about $22^{\circ} \mathrm{C}$ lower than that of PEI/PTA(3:7) ionic pair (about $48^{\circ} \mathrm{C}$ ). Like CA and HCA, DHCA would be able to accumulate as the pendants of the PEI chain of PEI/PTA(3/7) ionic pair through its hydrophobic interaction with the phenyl group of PTA and/or its electrostatic interaction with the PEI chain. Upon binding to the PEI chain as pendants, DHCA would be able to render the IPSAM disassembled at a lower temperature because the aromatic group of DHCA (dihydroxy phenyl group) is more polar than that of PTA (phenyl group).

DHCA was more effective than HCA in reducing the UCST of PEI/PTA(3/7) ionic pair. For example, the UCST of PEI/PTA/DHCA(3:7:1.4) ionic pair was about $12^{\circ} \mathrm{C}$ lower than that of PEI/PTA/HCA(3:7:1.4) ionic pair (about $39^{\circ} \mathrm{C}$ ). Since the aromatic group of DHCA (dihydroxy phenyl group) is more polar than that of HCA (hydroxyl phenyl group) $[25,26]$, the former phenolic acid would be able to decrease the UCST more effectively than the latter one. 
The UCST disappeared in the temperature window when the DHCA content increased furthermore. For example, the suspension of PEI/PTA/DHCA(3/7/4.2) ionic pair and that of PEI/PTA/DHCA(3/7/7) ionic pair were transparent in the full range of temperature tested. As the DHCA content is higher, the pendants of the PEI chains are likely to become more polar, thus the IPSAM would become more susceptible to the temperature increase, leading to a lower UCST.

In summary, PEI/PTA(3/7) ionic pair showed a UCST behavior and the phenolic acids decreased the UCST effective in the order of DHCA > HCA > CA. The phenolic acids were thought to accumulate as the pendants of the PEI chain of PEI/PTA(3/7) ionic pair through their hydrophobic interaction with the phenyl group of PTA and/or their electrostatic interaction with the PEI chain.

\subsection{Interfacial tensiometry}

Figure $3(\mathrm{~A})$ shows the interfacial tension of PEl solution, PTA solution, PEI/PTA(3/7) suspension, PEI/PTA/CA(3/7/C). PEl solution exhibited almost constant interfacial tension (ca. 72.3 dyne/cm) in a full range of concentrations tested. PEl is water-soluble and is known to be surface-inactive. PTA solution showed a decreasing profile in its interfacial tension in a saturation manner with increasing concentration. The interfacial tension of PTA solution decreased to $56 \mathrm{dyne} / \mathrm{cm}$ while the concentration increased up to $9 \mathrm{mg} / \mathrm{ml}$. PTA has a hydrophobic phenyl group and a hydrophilic carboxyl group thus it is an amphiphilic and surface-active molecule.

The surface tension of PEI/PTA(3/7) suspension also decreased in a saturation manner. It decreased more rapidly than that of PTA solution, indicating that PEI/PTA(3/7) ionic pair was more surface active than PTA. As described previously, PTA molecules would act as hydrophobic tails owing to their phenyl groups and a PEI chain would play a role as a hydrophilic head due to its polar property, rendering PEI/PTA(3/7) ionic pair surface-active. Since the lipophilic phenyl groups of PTA molecules can be adequately balanced by the hydrophilic PEI chain, the PEI/PTA ionic pair could be more surface active than PTA alone.

The interfacial tension profile of PEI/PTA/CA(3/7/1.4) suspension was almost the same as that of PEI/PTA(3/7) suspension. As described previously, CA would be able to accumulate as pendants of a PEI chain through its electrostatic interaction with the cationic polymer and/or its hydrophobic interaction with PTA (Fig. 5 (B)). However, CA had little effect on the interfacial tension of PEI/PTA(7/3) suspension, indicating that it hardly affected the interfacial activity. Even if CA is more polar than PTA, it seemed not to be polar enough to change the hydrophilic and lipophilic balance property of PEI/PTA(7/3) ionic pair. Further increase in the CA content could hardly alter the interfacial tension profile of PEI/PTA(7/3) suspension. For example, the interfacial tension profile of PEI/PTA/CA(3/7/4.2) and PEI/PTA/CA(3/7/7) suspension did not deviate markedly from that of PEI/PTA(7/3) suspension.

Figure 3 (B) shows the interfacial tension of PEI/PTA/HCA(3/7/c) suspension. The interfacial tension of $\mathrm{PEI} / \mathrm{PTA} / \mathrm{HCA}(3 / 7 / 1.4)$ suspension was higher than that of PEI/PTA(3/7) suspension in the full range of concentration tested. 
If HCA accumulates as pendants of a PEl chain, the hydrophobicity of the pending groups of PEI/PTA(3/7) ionic pair would decrease because the hydroxyphenyl group of HCA is more polar than the phenyl group of PTA. Accordingly, the hydrophilic and lipophilic balance could be broken by the attachment of HCA to PEI chains, leading to a decrease in the interfacial activity of PEI/PTA(3/7) ionic pair. As the HCA content increased, the interfacial tension increased. For example, the minimum interfacial tension of PEI/PTA/HCA(3/7/1.4), PEI/PTA/HCA(3/7/4.2), and PEI/PTA/HCA(3/7/7) suspension was $52.9,53.8$, and 54.3 dyne/cm, respectively. That is, the interfacial activity of $\mathrm{PEI} / \mathrm{PTA} / \mathrm{HCA}(3 / 7)$ ionic pair decreased with increasing the HCA content.

Figure 3 (C) shows the interfacial tension of PEI/PTA/DHCA (3/7/C) suspension. As PEI/PTA/HCA(3/7/1.4) suspension did, PEI/PTA/DHCA(3/7/1.4) suspension exhibited higher interfacial tensions than PEI/PTA(3/7) suspension in the full range of concentration tested. If DHCA accumulates as pendants of a PEI chain, PEI/PTA(3/7) ionic pair would decrease in its interfacial activity because the dihydroxyphenyl group of DHCA is more polar than the phenyl group of PTA. As PEI/PTA/HCA $(a / b / c)$ suspension did, PEI/PTA/DHCA(a/b/c) suspension showed an increasing interfacial activity with decreasing the DHCA content. For example, the minimum interfacial tension of PEI/PTA/DHCA (3/7/1.4), PEI/PTA/DHCA(3/7/4.2), and PEI/PTA/DHCA(3/7/7) suspension was 53.5, 54.5, and $55.2 \mathrm{dyne} / \mathrm{cm}$, respectively. That is, the interfacial activity of PEI/PTA(3/7) ionic pair decreased with increasing the DHCA content. In case of both PEI/PTA/HCA $(\mathrm{a} / \mathrm{b} / \mathrm{c})$ and PEI/PTA/DHCA(a/b/c) suspension, however, the interfacial tension increased in a saturation manner but not as much as the phenolic acids (i.e. HCA and DHCA) content increased. This is possible because, once the binding site of PEI/PTA(3/7) ionic pair is saturated by the phenolic acids, the further attachment of the phenolic acid would hardly change the interfacial property of PEI/PTA(3/7) ionic pair.

In summary, PEI/PTA(3/7) ionic pair was found to be air/water interface-active due to their amphiphilic property. The phenolic acids (i.e. CA. HCA, and DHCA) would be able to accumulate as pendants of a PEI chain through its electrostatic interaction with the cationic polymer and/or its hydrophobic interaction with PTA. However, CA had little effect on the interfacial activity of the ionic pair, because the difference in polarity between PTA and CA was not much enough to affect the hydrophilic and lipophilic balance property of PEI/PTA(7/3) ionic pair. HCA and HDCA decreased the interfacial activity possibly because they are more polar than $\mathrm{CA}$ and the hydrophilic and lipophilic balance could be broken by their attachment to PEl chains.

\subsection{Transmission electron microscopy}

Figure 4 (A) shows the TEM micrograph of PEI/PTA(3/7) and PEI/PTA/CA(3/7/c) suspension. Circular objects whose diameter ranged from 20-90nm were found with all the suspensions examined and they were believed to be IPSAM. Since the suspensions were dried at room temperature $\left(20-23^{\circ} \mathrm{C}\right)$ which was far below the UCST of PEI/PTA(3/7) ionic pair and that of PEI/PTA/CA(a/b/c) ionic pair (Fig. $2(\mathrm{~A})$ ), the suspensions would contain the ionic pairs as a form of IPSAM. 
Figure 4 (B) shows the TEM micrograph of PEI/PTA/HCA(3/7/C) suspension. IPSAM was found with PEI/PTA/HCA(3/7/1.4) and PEI/PTA/HCA(3/7/4.2), but not with PEI/PTA/HCA(3/7/7) suspension. In fact, PEI/PTA/HCA (3/7/1.4) ionic pair exhibited its UCST far above room temperature, and PEI/PTA/HCA $(3 / 7 / 4.2)$ ionic pair showed its UCST below room temperature but still underwent the phase transition above room temperature (Fig. 2 (B)). Thus, PEI/PTA/HCA(3/7/1.4) and PEI/PTA/HCA(3/7/4.2) suspension were able to contain IPSAM at room temperature, shown in Fig. 4 (B). However, the UCST behavior could hardly be found with PEI/PTA/HCA(3/7/7) ionic pair in 20 to $50^{\circ} \mathrm{C}$ and the phase transition temperature seemed to be far below room temperature (Fig. 2 (B)). As a result, PEI/PTA/HCA(3/7/7) suspension could hardly contain IPSAM at room temperature, shown in Fig. 4 (B).

Figure 4 (C) shows the TEM micrograph of PEI/PTA/DHCA(3/7/C) suspension. PEI/PTA/DHCA(3/7/1.4) suspension showed IPSAM but not PEI/PTA/DHCA(3/7/4.2) and PEI/PTA/DHCA(3/7/7) suspension. PEI/PTA/DHCA (3/7/1.4) ionic pair exhibited its UCST above room temperature (Fig. 2 (C)) thus its suspension could include IPSAM at room temperature, shown in Fig. 4 (C). PEI/PTA/DHCA(3/7/4.2) and PEI/PTA/DHCA(3/7/7) ionic pair did not show any UCST behavior in the full range of temperature tested (i.e. 20 to $50^{\circ} \mathrm{C}$ ) and its phase transition temperature seemed to be far below room temperature (Fig. 2 (C)). Accordingly, the suspensions were hardly able to contain IPSAM at room temperature, shown in Fig. 4 (C).

In summary, IPSAM was found as nanoparticles whose diameter was tens of nanometer and PA had little effect on the shape and the size of IPSAM, once the UCST of PEI/PTA/PA(a/b/c) ionic pair was above room temperature $\left(20-23^{\circ} \mathrm{C}\right)$ where the TEM micrographs were taken. If the UCST of PEI/PTA/PA $(a / b / c)$ ionic pair was below room temperature, no particulate matters were found on the TEM micrographs.

\subsection{Investigation of PEI/PTA/PA interaction by ${ }^{1} \mathrm{H}$ NMR spectroscopy}

Figure 5 (A) shows the ${ }^{1} \mathrm{H}$ NMR spectrum of PEI, PTA, and PEI/PTA(3/7) ion pair. In the spectrum of PTA, the phenyl group was found around $7.45 \mathrm{ppm}$. In the spectrum of PEI/PTA(3/7) ion pair, the phenyl group shifted upfield and it was found around $7.38 \mathrm{ppm}$. The chemical shift was possible because the carboxyl group of PTA could ionically interact with the amino group of PEI. PEI/PTA ion pairs could compose IPSAM in an aqueous solution due to their amphiphilic property.

Figure $5(B)$ shows the ${ }^{1} \mathrm{H}$ NMR spectrum of CA, PEI/PTA(3/7) ion pair, and PEI/PTA/CA(3/7/1.4) ion pair. In the spectrum of PEI/PTA(3/7) ion pair, the phenyl group was found around $7.38 \mathrm{ppm}$. In the spectrum of CA, the phenyl group was found around $7.64 \mathrm{ppm}$. Compared with the spectrum of PEI/PTA(3/7) ion pair and CA, a peak was newly found around $7.75 \mathrm{ppm}$ in the spectrum of PEI/PTA/CA(3/7/1.4) ion pair. This suggested that the signal of the phenyl group shifted downfield from 7.64 to $7.75 \mathrm{ppm}$ by the addition of CA to PEI/PTA(3/7) ion pair. As described previously, CA would be able to bind to PTA through the hydrophobic interaction among the phenyl groups. The hydrophobic interaction would be responsible for the chemical shift. 
Figure 5 (C) shows the ${ }^{1} \mathrm{H}$ NMR spectrum of HCA, PEI/PTA(3/7) ion pair, and PEI/PTA/HCA(3/7/1.4) ion pair. The phenyl group of HCA was found around 7.56 and $6.90 \mathrm{ppm}$, while the phenyl group of PEI/PTA/HCA(3/7/1.4) ion pair shifted downfield and found around 7.71 and 7.00 ppm. Like CA, HCA would bind to PTA through the hydrophobic interaction among the phenyl groups, causing the chemical shift.

Figure 5 (D) shows the ${ }^{1} \mathrm{H}$ NMR spectrum of DHCA, PEI/PTA(3/7) ion pair, and PEI/PTA/DHCA(3/7/1.4) ion pair. In the spectrum of PEI/PTA(3/7) ion pair, the phenyl group was found around $7.38 \mathrm{ppm}$. In the spectrum of DHCA, the phenyl group was found around 7.17 to $6.94 \mathrm{ppm}$. Compared with the spectrum of DHCA and PEI/PTA(3/7), a new peak was found around $7.64 \mathrm{ppm}$ in the spectrum of PEI/PTA/DHCA(3/7/1.4) ion pair, suggesting that the signal of the phenyl group shifted downfield. Like CA and HCA, DHCA could also bind to PTA through the hydrophobic interaction, leading to the downfield shift.

In summary, upon the addition of PA to PEI/PTA ion pairs, the signal of phenyl group shifted downfield on the $1 \mathrm{H}$ NMR spectrum. The chemical shift would be caused by the hydrophobic interaction of the phenyl group of PA (i.e. CA, HCA, and DHCA) with that of PTA.

\subsection{Temperature-responsive release}

Figure 6 (A) shows the temperature-dependent release profile of dye loaded in PEI/PTA(3/7) IPSAM. No substantial release was found when the temperature of release medium was 24 and $30^{\circ} \mathrm{C}$, where the maximum release degree was only about 3 and $4 \%$, respectively. Since the UCST of PEI/PTA(3/7) ionic pair was about $48^{\circ} \mathrm{C}$, the IPSAM were thought to be stable and could maintain its integrity at such low temperatures. When the temperature was 37 and $45^{\circ} \mathrm{C}$, the release took place appreciably and the maximum release degree was about $8 \%$ and $9.5 \%$. The temperature of the release medium (i.e. 37 and $45^{\circ} \mathrm{C}$ ) was still below the UCST. However, the IPSAM would be able to be fluidized due to the thermally promoted vibration of the PEI/PTA ionic pair at such high temperatures. In addition, the diffusivity of dye loaded in the IPSAM would be greater when the temperature was higher $[27,28]$. This could account for why the substantial release was observed at 37 and $45^{\circ} \mathrm{C}$ despite the temperature was below the UCST.

Figure 6 (B) shows the temperature-dependent release profile of dye loaded in PEI/PTA/CA(3/7/1.4) IPSAM. The temperature-dependent release profiles of dye loaded in PEI/PTA/CA(3/7/1.4) IPSAM were similar to those of dye loaded in PEI/PTA(3/7) IPSAM. As PEI/PTA(3/7) IPSAM did, PEI/PTA/CA(3/7/1.4) IPSAM released its payload in proportion to the temperature. For example, the maximum release degree was about $7,7.5,11$, and $12.5 \%$, respectively, when the temperature was $24,30,37$, and $45^{\circ} \mathrm{C}$. The thermally induced fluidization of the IPSAM and/or the thermally promoted diffusion of the payload would be a reason for the increased release with temperature. However, ascent width in the release degree was not marked. This is possible because the temperature of release medium was not greater than the UCST of PEI/PTA/CA(3/7/1.4) IPSAM (i.e. $45^{\circ} \mathrm{C}$ ) thus the IPSAM could maintain its integrity. 
Figure 6 (C) shows the temperature-dependent release profile of dye loaded in PEI/PTA/HCA(3/7/1.4) IPSAM. The release degree increased gradually with time lapse when the temperature release medium was below the UCST of the IPSMA (ca. $39^{\circ} \mathrm{C}$ ) (i.e. $24,30,37^{\circ} \mathrm{C}$ ). The release degree increased gently with increasing temperature below the UCST. For example, the maximum release degree was about 7, 12.5, and $17 \%$, respectively, when the temperature was 24,30 , and $37^{\circ} \mathrm{C}$. On the other hand, the release degree increased rapidly in early stage and in a saturation manner in later stage, when the temperature was above the UCST (ca. $39^{\circ} \mathrm{C}$ ) (i.e. $45^{\circ} \mathrm{C}$ ). The release degree at $45^{\circ} \mathrm{C}$ was much higher than those observed below the UCST. For example, the maximum release degree at $45^{\circ} \mathrm{C}$ was about $40 \%$, far higher than those observed above the UCST $(7,12.5$, and $17 \%)$. The rapid and extensive release could be ascribed to the thermally-induced disintegration of the IPSAM. When the temperature of release medium was above UCST, the IPSAM would be disassembled and release its payload extensively.

Figure 6 (D) shows the temperature-dependent release profile of dye loaded in PEI/PTA/DHCA(3/7/1.4) IPSAM. The release degree increased slowly with time lapse and the maximum release degree was only about $10 \%$, when the temperature release medium was $24^{\circ} \mathrm{C}$, below the UCST of PEI/PTA/DHCA(3/7/1.4) ionic pair (ca. $\left.26^{\circ} \mathrm{C}\right)$. Whereas, when the temperature of release medium was above the UCST, the release degree increased rapidly with time lapse and the release degree was far higher than that observed below the UCST. For example, the maximum release degree at 30,37 , and $45^{\circ} \mathrm{C}$ were about $30 \%, 40 \%$, and $43.5 \%$, respectively, which were much higher than that observed below the UCST. The thermally-induced disintegration was thought to be responsible for the intensive release.

In summary, the release degree of cargo loaded in IPSAM increased slowly with time lapse below UCST. Whereas, the release degree increased rapidly with time lapse above UCST, possibly because of the thermally-induced disintegration of IPSAM.

\section{Conclusions}

Phenolic acids including CA, HCA, and DHCA were included in PEI/PTA mixture solution to investigate their effect on the physicochemical and thermal properties of PEI/PTA(3/7) ionic pair and IPSAM. The phenolic acids lowered the UCST effectively in the order of DHCA > HCA > CA. The phenolic acids were thought to attach to the PEI chain of the ionic pair through hydrophobic and/or electrostatic interactions, evidenced by FT-IR and ${ }^{1} \mathrm{H}$ NMR spectroscopy. CA hardly affected the interfacial activity of the ionic pair, possibly because $C A$ is not polar enough to affect the amphiphilic property of PEI/PTA(7/3) ionic pair. Whereas, HCA and HDCA significantly decreased the interfacial activity possibly because they are polar enough to affect the amphiphilic property of the ionic pair. IPSAM could be found on the TEM micrograph once the UCST of PEI/PTA/PA(a/b/c) ionic pair was above room temperature $\left(20-23^{\circ} \mathrm{C}\right)$. IPSAM seemed to be nanoparticles whose diameter was tens of nanometer and PA had little effect on the shape and the size of IPSAM, If the UCST of PEI/PTA/PA(a/b/c) ionic pair was below room temperature, no particulate matters could be found on the TEM micrograph. Below the UCST, the release degree of cargo loaded in IPSAM increased slowly with time lapse. Whereas, above the UCST, it increased rapidly with time lapse, possibly because of the thermally-induced disintegration of IPSAM.

Page $12 / 22$ 
IPSAM prepared in this study would be applicable to the development of a drug carrier that can release its payload in a temperature-responsive manner.

\section{Declarations}

Consent for publication: All authors consent for publication.

Availability of data and materials: All data are fully available without restriction.

Conflicts of interest: The authors declare no conflict of interest.

\section{Funding}

This research was supported by Basic Science Research Program through the National Research Foundation of Korea (NRF) funded by the Ministry of Education(No. 2018R1A6A1A03025582) and the Basic Science Research Program through the National Research Foundation of Korea (NRF) funded by the Ministry of Education (NRF-2018R1D1A1B07043439).

Authors' contributions: JCK conceptualized and supervised the study. FZ performed the experiments. JCK and FZ analyzed the data and wrote the manuscript.

Acknowledgements: Not applicable.

\section{References}

[1] Sorrenti A, Illa O, Ortuño R M. Amphiphiles in aqueous solution: well beyond a soap bubble. Chem Soc Rev. 2013, 42: 8200-8219.

[2] Wang C, Wang Z, Zhang X. Amphiphilic building blocks for self-assembly: from amphiphiles to supraamphiphiles. Acc Chem Res. 2012, 45: 608-618.

[3] van't Hag L, Gras S L, Conn C E, et al. Lyotropic liquid crystal engineering moving beyond binary compositional space-ordered nanostructured amphiphile self-assembly materials by design. Chem Soc Rev. 2017, 46: 2705-2731.

[4] $\mathrm{Ma} \mathrm{H}, \mathrm{Hao}$ J. Ordered patterns and structures via interfacial self-assembly: superlattices, honeycomb structures and coffee rings. Chemical Society Reviews. 2011, 40: 5457-5471.

[5] Nagarajan R. Molecular packing parameter and surfactant self-assembly: the neglected role of the surfactant tail. Langmuir. 2002, 18: 31-38.

[6] Lichtenberg D, Ahyayauch $\mathrm{H}$, Alonso A, et al. Detergent solubilization of lipid bilayers: a balance of driving forces. Trends Biochem Sci. 2013, 38: 85-93. 
[7] Komaiko J S, McClements D J. Formation of food-grade nanoemulsions using low-energy preparation methods: A review of available methods. Compr Rev Food Sci Food Saf. 2016, 15: 331-352.

[8] Zhao Y, Yokoi H, Tanaka M, et al. Self-assembled pH-responsive hydrogels composed of the RATEA16 peptide. Biomacromolecules. 2008, 9: 1511-1518.

[9] Wang C, Kang Y, Liu K, et al. pH and enzymatic double-stimuli responsive multi-compartment micelles from supra-amphiphilic polymers. Polym Chem. 2012, 3: 3056-3059.

[10] Jiang F, Chen S, Cao Z, et al. A photo, temperature, and pH responsive spiropyran-functionalized polymer: Synthesis, self-assembly and controlled release. Polymer. 2016, 83: 85-91.

[11] Yang F, Cao Z, Wang G. Micellar assembly of a photo-and temperature-responsive amphiphilic block copolymer for controlled release. Polym Chem. 2015, 6: 7995-8002.

[12] Kim J C, Bae S K, Kim J D. Temperature-sensitivity of liposomal lipid bilayers mixed with poly (Nisopropylacrylamide-co-acrylic acid). J Biochem. 1997, 121: 15-19.

[13] Nakahata M, Takashima Y, Harada A. Redox-responsive macroscopic gel assembly based on discrete dual interactions.Angew. Chem Int Ed. 2014, 53: 3617-3621.

[14] Zuo C, Dai X, Zhao S, et al. Fabrication of dual-redox responsive supramolecular copolymers using a reducible $\beta$-cyclodextran-ferrocene double-head unit. ACS Macro Lett. 2016, 5: 873-878.

[15] Murdan S. Electro-responsive drug delivery from hydrogels. J Control Release. 2003, 92: 1-17.

[16] Lee S J, Jeong J R, Shin S C, et al. Magnetic enhancement of iron oxide nanoparticles encapsulated with poly (D, L-latide-co-glycolide). Colloids Surf A Physicochem Eng Asp. 2005, 255: 19-25.

[17] Park D, Kim J C. UV light and thermo-sensitive disassembling and release property of the assembly of cinnamic acid and poly (ethyleneimine). Soft Mater. 2017, 15: 282-291.

[18] Guo H, Kim J C. Upper critical solution temperature behavior of cinnamic acid and polyethyleneimine mixture and its effect on temperature-dependent release of liposome. Int J Pharm. 201, 494: 172-179.

[19] Kim T H, Alle M, Park S C, et al. Self-assembly prepared using an ion pair of poly (ethylene imine) and (phenylthio) acetic acid as a drug carrier for oxidation, temperature, and NIR-responsive release. Chem Eng J. 2021, 415: 128954.

[20] Kim T H, Alle M, Kim J C. Oxidation-and Temperature-Responsive Poly (hydroxyethyl acrylate-cophenyl vinyl sulfide) Micelle as a Potential Anticancer Drug Carrier. Pharmaceutics. 2019, 11: 462.

[21] Chen W, Zhang C, Song L, et al. A high throughput Nile red method for quantitative measurement of neutral lipids in microalgae. J Microbiol Methods. 2009, 77: 41-47. 
[22] Larsson K., Fontell K, and Krog N. Structural relationships between lamellar, cubic and hexagonal phases in monoglyceride-water systems. Possibility of cubic structures in biological systems. Chem Phys Lipids. 1980, 27: 321-328.

[23] Amara $\mathrm{M}$, Kerdjoudj $\mathrm{H}$. Modification of the cation exchange resin properties by impregnation in polyethyleneimine solutions: application to the separation of metallic ions. Talanta. 2003, 60: 991-1001.

[24] Pettit L D, Royston A, Sherrington C, et al. The acid dissociation constants of substituted (phenylthio) acetic and (phenylseleno) acetic acids. J Chem Soc B Phys Org. 1968, 588-590.

[25] Shinoda K, Carlsson A, Lindman B. On the importance of hydroxyl groups in the polar head-group of nonionic surfactants and membrane lipids. Adv Colloid Interface Sci. 1996, 64: 253-271.

[26] 3.4: Functional Groups [Internet]. [uplated 2019 April 28; cited 2021 August 23]. Available from: https://bio.libretexts.org/@go/page/8390

[27] Stephenson J C, Wood R E, Moore C B. (1971). Temperature Dependence of Intramolecular Vibration $\rightarrow$ Vibration Energy Transfer in CO2. J Chem Phys. 1971, 54: 3097-3102.

[28] Jacobs M H. Diffusion processes. In Diffusion Processes. Springer, 1935. p. 1-145.

\section{Figures}



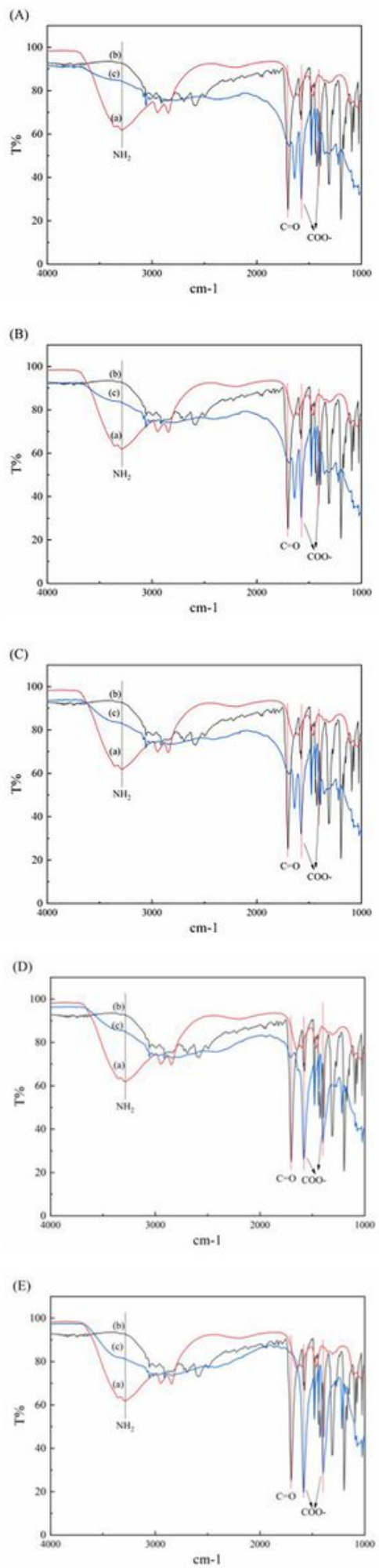

Figure 1

FT-IR spectrum of PEI (a, red), PTA (b, black), and dry PEI/PTA(3/7) (c, blue). The molar ratio of PEI/PTA is 3:7 (A), 4:6 (B), 5:5 (C), 6:4 (D), 7:3 (E). 

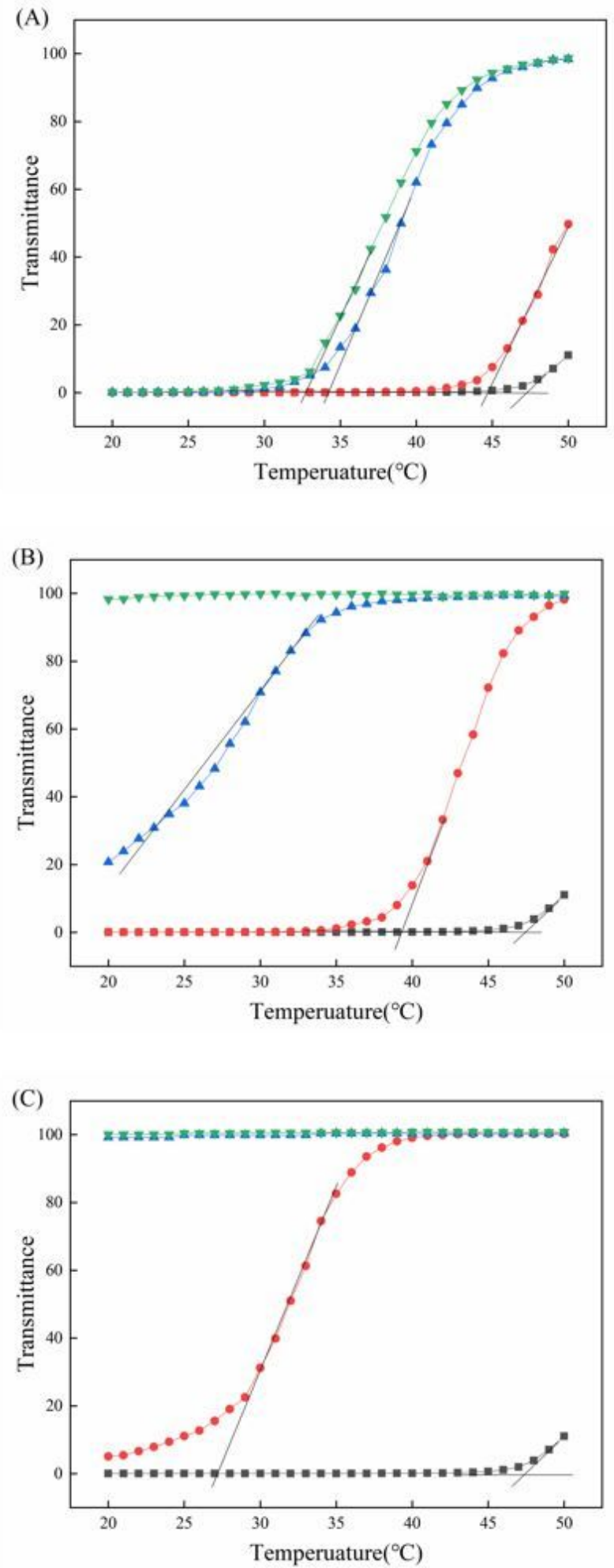

Figure 2

Transmittance of PEI/PTA/CA(3/7/C) suspension (A), PEI/PTA/HCA(3/7/c) suspension (B), and $\mathrm{PEI} / \mathrm{PTA} / \mathrm{DHCA}(3 / 7 / \mathrm{c})$ suspension $(\mathrm{C})$ with increasing temperature. $\mathrm{c}=0$ ( $\boldsymbol{\square}$, black), 1.4( $\boldsymbol{\bullet}$, red), 4.2( $\boldsymbol{\Delta}$, blue) and $7(\boldsymbol{\nabla}$, green $)$. 

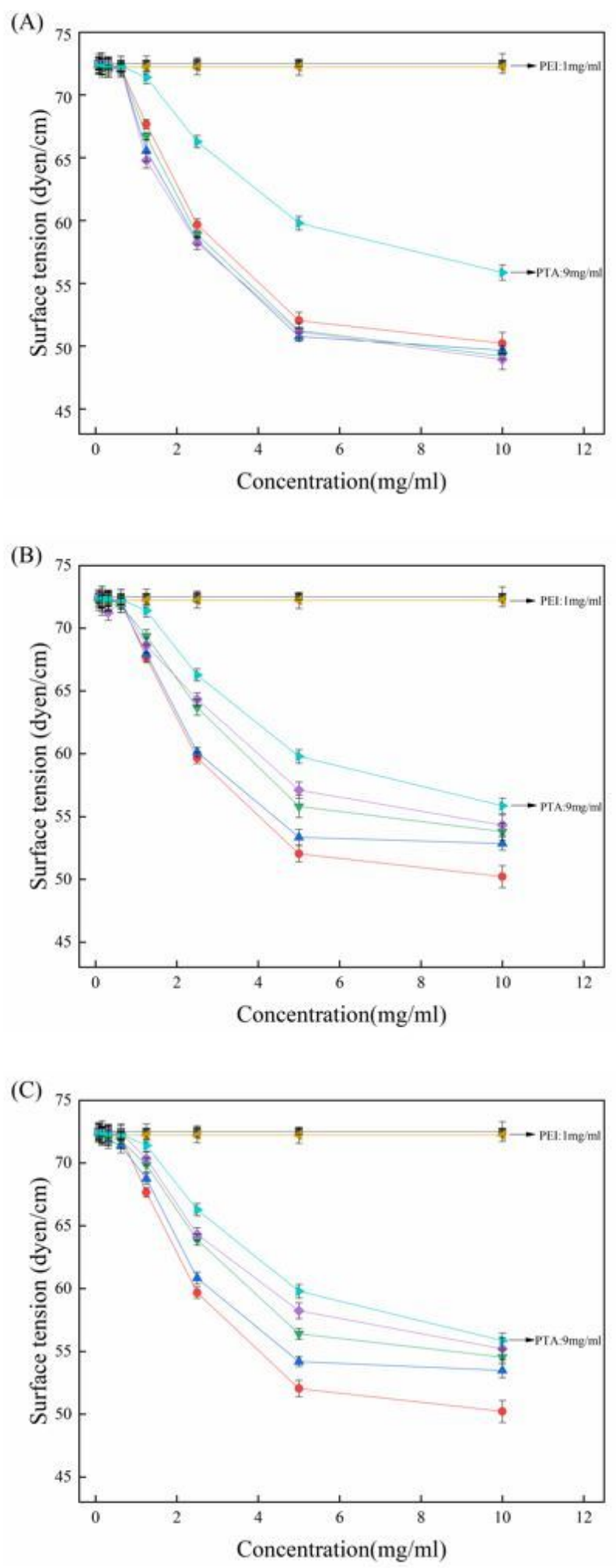

\section{Figure 3}

Interfacial tension of Trizma buffer $(\square$, black), PEI solution ( $\triangleleft$, yellow), PTA solution $(\bullet$, azure), PEI/PTA(3/7) (O, red), PEI/PTA/CA (3/7/c) suspension (A), PEI/PTA/HCA (3/7/c) suspension (B), and

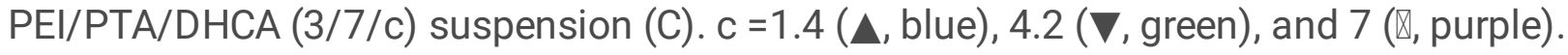


(A)
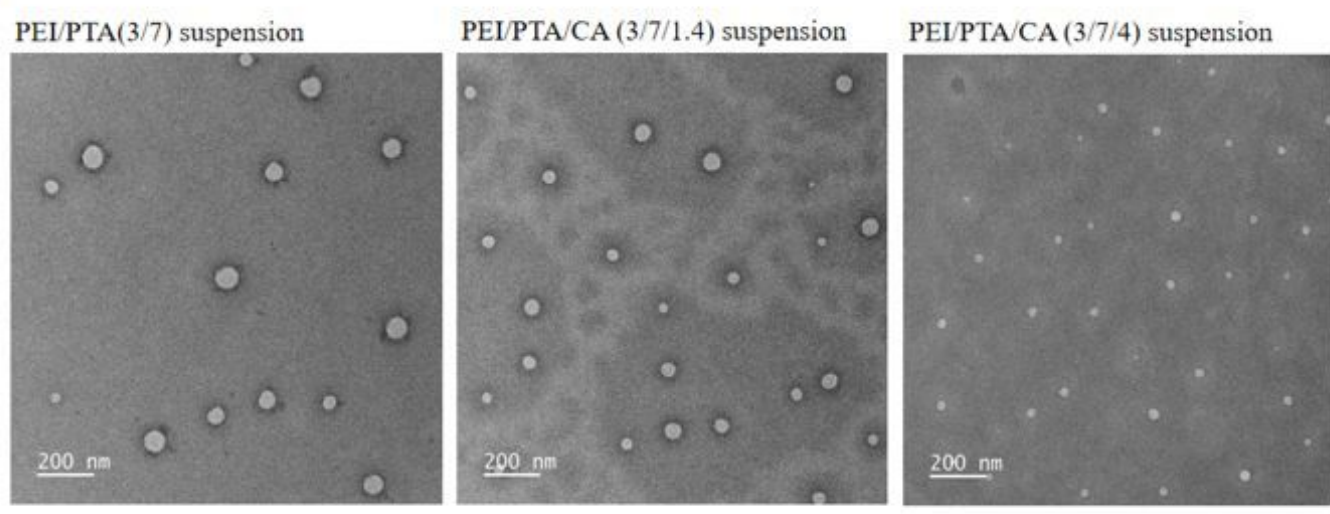

PEI/PTA/HCA (3/7/7) suspension

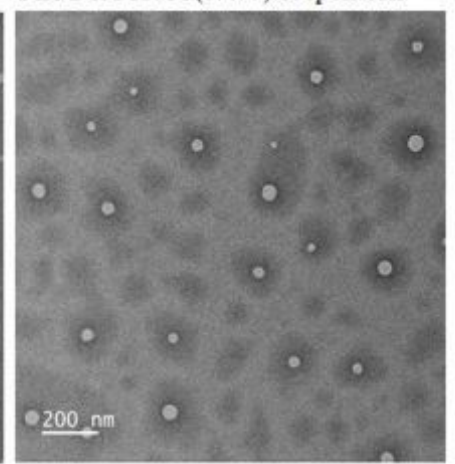

(B)
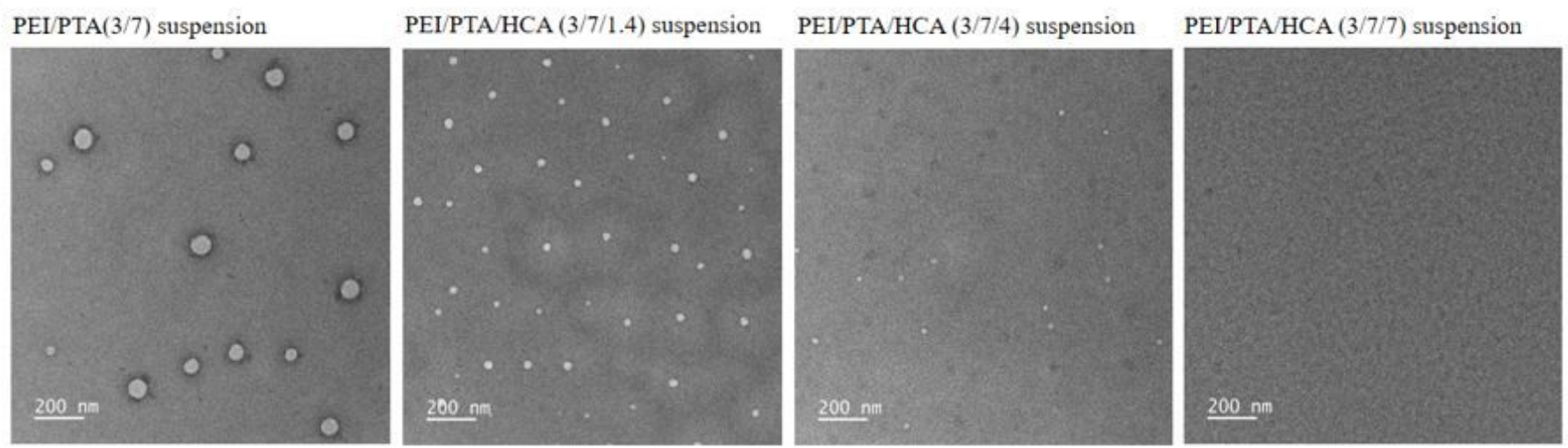

(C)

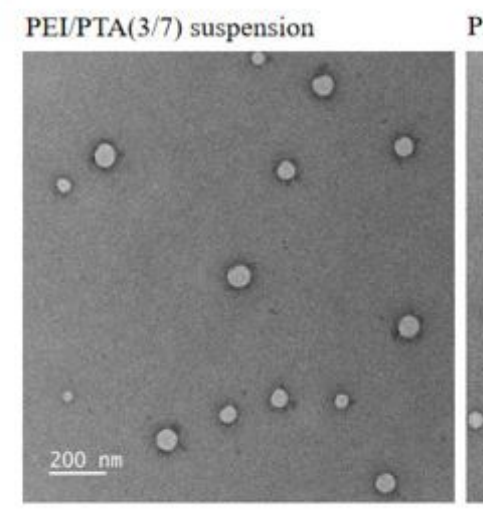
PEI/PTA/DHCA (3/7/1.4) suspension PEI/PTA/DHCA (3/7/4) suspension
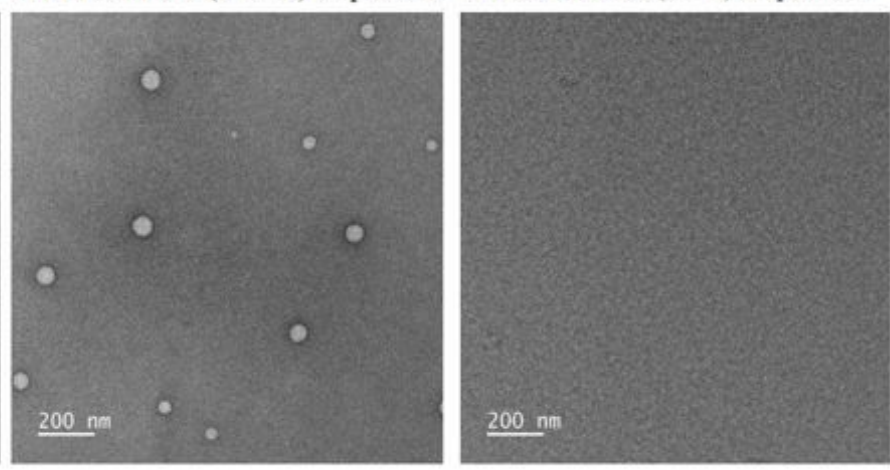

PEI/PTA/DHCA (3/7/7) suspension

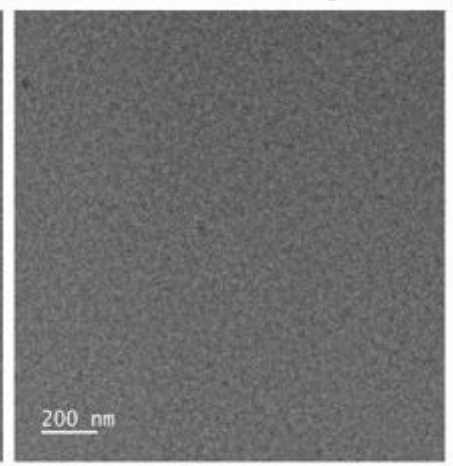

\section{Figure 4}

TEM micrograph of PEI/PTA(3/7), PEI/PTA/CA (3/7/c) suspension (A), PEI/PTA/HCA (3/7/c) suspension (B), and PEI/PTA/DHCA (3/7/C) suspension (C). $\mathrm{C}=1.4,4.2$, and 7. 
(A)
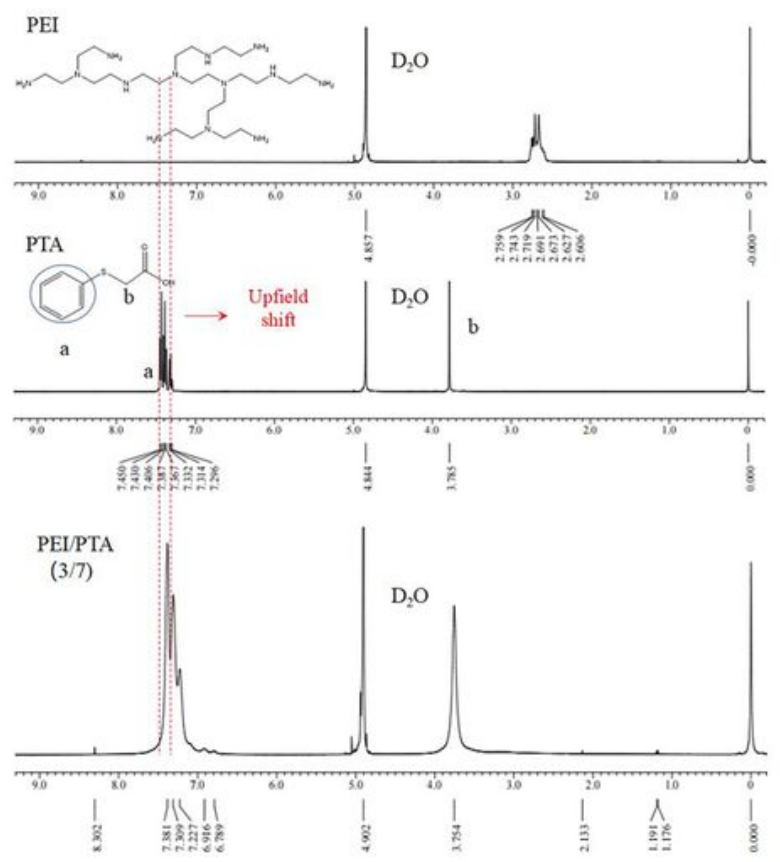

(B)
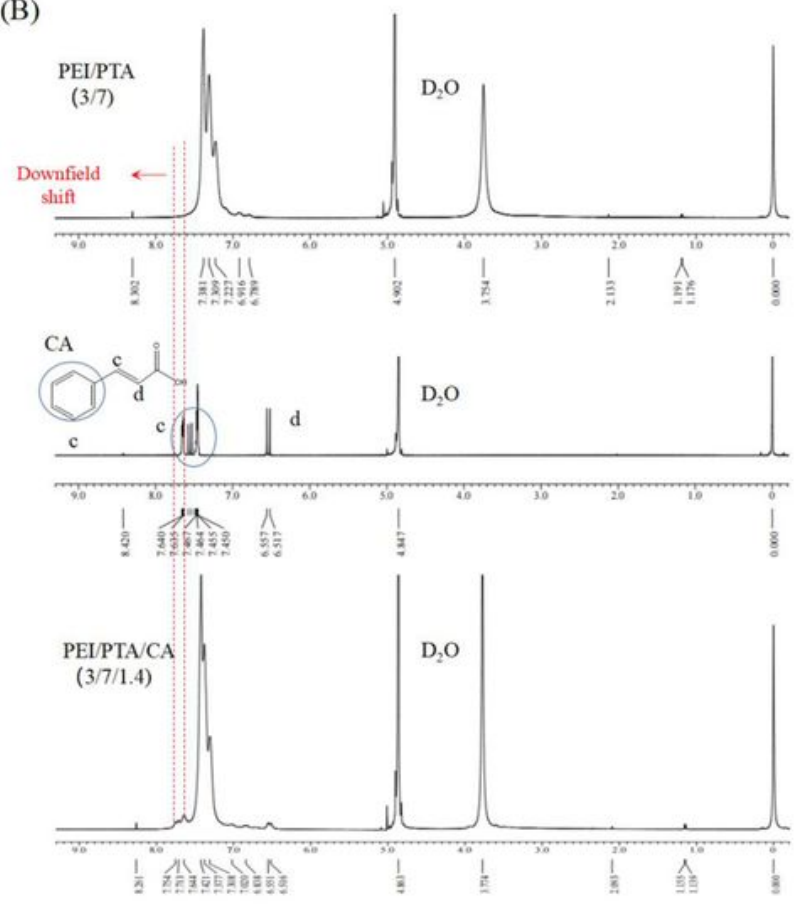

(C)
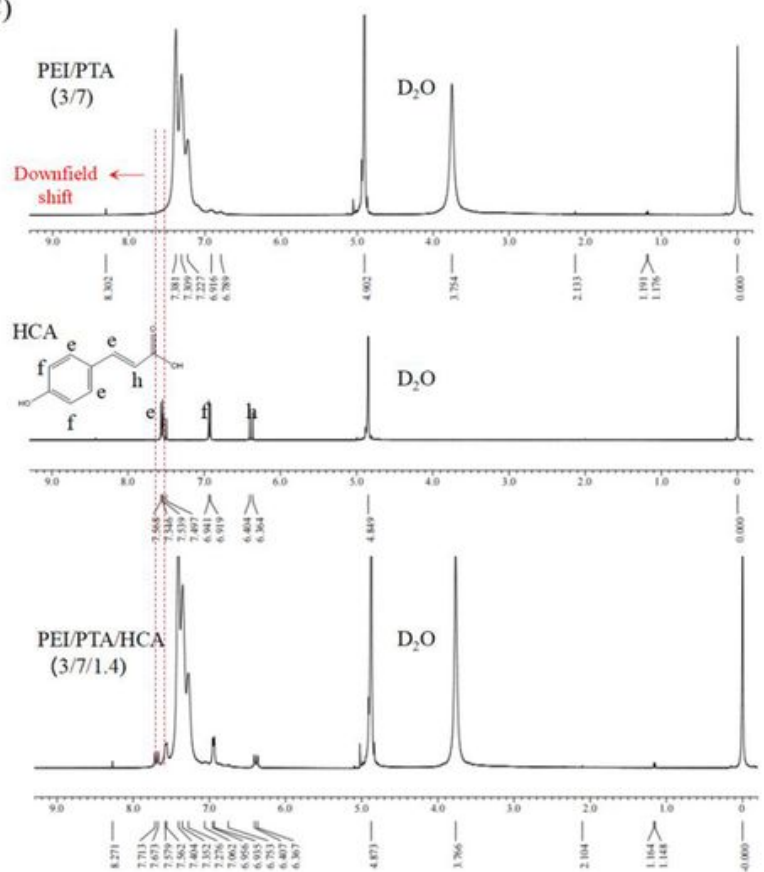

(D)
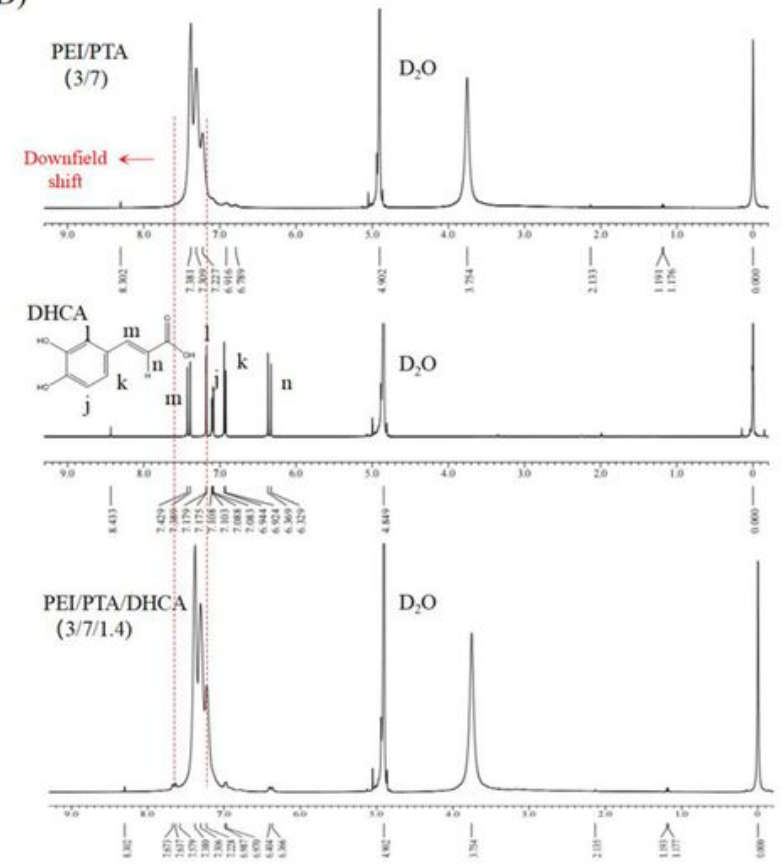

\section{Figure 5}

1H NMR spectrum of PEI, PTA, PEI/PTA(3/7) (A), CA, PEI/PTA(3/7), PEI/PTA/CA(3/7/1.4) (B), HCA, PEI/PTA(3/7), PEI/PTA/HCA(3/7/1.4) (C), DHCA, PEI/PTA(3/7), PEI/PTA/DHCA(3/7/1.4) (D). 

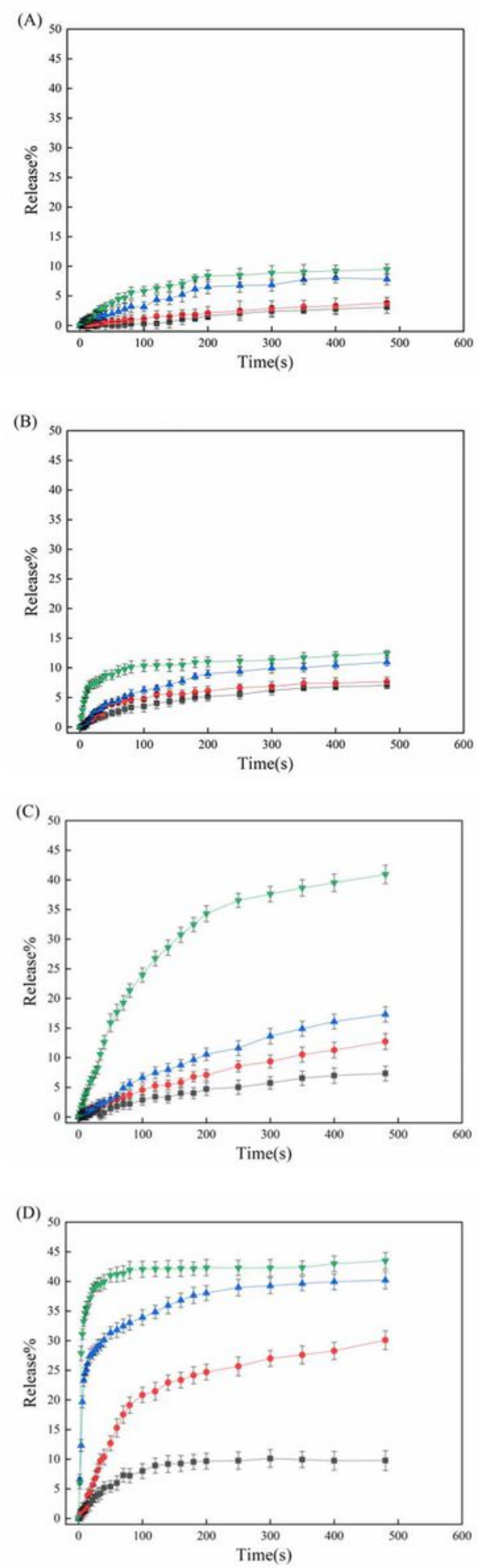

Figure 6

Temperature-dependent release profile of dye loaded in PEI/PTA(3/7) IPSAM (A), PEI/PTA/CA(3/7/1.4) IPSAM (B), PEI/PTA/HCA(3/7/1.4) IPSAM (C), and PEI/PTA/DHCA(3/7/1.4) IPSAM (D) at 24 ( $\boldsymbol{\square}$, black), $30(\bullet$, red $), 37(\boldsymbol{\Lambda}$, blue $)$ and $45^{\circ} \mathrm{C}(\boldsymbol{\nabla}$, green $)$.

\section{Supplementary Files}


This is a list of supplementary files associated with this preprint. Click to download.

- GraphicalAbstract.tif 